\title{
An alternative method for phase-unwrapping of interferometric data
}

\author{
E. de la Rosa-Miranda \\ E. Gonzalez-Ramirez \\ gonzalez_efren@hotmail.com
}

J. J. Villa-Hernández jvillah@yahoo.com

L. R. Berriel-Valdos

berval@inaoep.mx

\section{Olvera-Olvera}

\section{J. I. de la Rosa-Vargas ismaelrv@yahoo.com}

\section{T. Saucedo-Anaya} tsaucedo@fisica.uaz.edu.mx

\section{J. G. Arceo Olague}

\section{Alaniz-Lumbreras}

\section{M. Castaño}

meneses@unam.mx

\author{
Unidad Académica de Ingeniería Eléctrica, Universidad Autónoma de Zacatecas, Antiguo Camino a la \\ Bufa No. 1, Col. Centro. C. P. 98000, Zacatecas, Zac. México \\ Unidad Académica de Ingeniería Eléctrica, Universidad Autónoma de Zacatecas, Antiguo Camino a la \\ Bufa No. 1, Col. Centro. C. P. 98000, Zacatecas, Zac. México \\ Unidad Académica de Ingeniería Eléctrica, Universidad Autónoma de Zacatecas, Antiguo Camino a la \\ Bufa No. 1, Col. Centro. C. P. 98000, Zacatecas, Zac. México \\ Luis Enrique Erro No. 1, Santa María Tonantzintla, San Andrés Cholula, C.P. 72840, Puebla, México
}

Unidad Académica de Ingeniería Eléctrica, Universidad Autónoma de Zacatecas, Antiguo Camino a la Bufa No. 1, Col. Centro. C. P. 98000, Zacatecas, Zac. México

Unidad Académica de Ingeniería Eléctrica, Universidad Autónoma de Zacatecas, Antiguo Camino a la Bufa No. 1, Col. Centro. C. P. 98000, Zacatecas, Zac. México

Unidad Académica de Física, Universidad Autónoma de Zacatecas, Calzada Solidaridad Esquina con Paseo a la Bufa $\backslash \backslash$ N. Col. Centro. C. P. 98000, Zacatecas, Zac. México

Unidad Académica de Ingeniería Eléctrica, Universidad Autónoma de Zacatecas, Antiguo Camino a la Bufa No. 1, Col. Centro. C. P. 98000, Zacatecas, Zac. México

Unidad Académica de Ingeniería Eléctrica, Universidad Autónoma de Zacatecas, Antiguo Camino a la Bufa No. 1, Col. Centro. C. P. 98000, Zacatecas, Zac. México

CIATEQ Unidad Querétaro, México, On sabbatical leave from Centro de Física Aplicada y Tecnología Avanzada, Universidad Nacional Autónoma de México

In this paper we present a novel algorithm for phase unwrapping where only a subset of data from the wrapped phase map is used to reconstruct the unwrapped phase map as a linear combination of radial basis functions (RBF's). For noisy phase maps this algorithm gives better results than three reference algorithms based on radial basis functions, Zernike polynomials and path dependent phase unwrapping strategies.

[DOI: http://dx.doi.org/10.2971/jeos.2014.14040]

Keywords: Phase unwrapping, radial basis functions

\section{I NTRODUCTION}

Interferometric methods are widely used to measure physical magnitudes such as deformation, stress, temperature, etc. $[1,2]$ in a non destructive and non invasive way. These magnitudes modulate a fringe pattern called interferogram which contains the information about the related physical magnitude. Demodulation is necessary to recover the phase information related to these magnitudes.

Techniques for phase recovery such as Fourier based [3], phase stepping [4] or regularization [5]-[7], provide a noncontinuous phase wrapped in the interval $(-\pi, \pi]$. This phase must be unwrapped to obtain the physical magnitudes. It is common to find phase inconsistencies or noise that can make the unwrapping process a difficult task. The application of path dependent algorithms [8] improves the unwrapping process but does not always provide proper results, Ghiglia et al. shows a simple test for path dependence [9]. A robust alternative for many cases is the least-squares solution which is described in matrix form by Hunt [10]. Another robust algorithm to find a solution in the presence of path-integral phase inconsistencies using the cosine transform is that proposed by Ghiglia and Romero [11]. The methods above mentioned require long processing time and are computationally complex that make them inconvenient for some applications. When the phase is smooth, the time of processing can be shortened by solving the phase unwrapping problem using a linear combination of local basis functions [12]. Moreover, Arines' method [13] uses a linear combination of Zernike polynomials to reconstruct any smooth phase map. These polynomials are utilized in functions with circular domain. In this paper we propose a regular grid of a linear combination of gaussian basis functions (RBF's) applied to unwrap the phase. The algorithm proposed here takes into account part of the phase map, using only the image pixels forming a regular mesh. This strategy is important to reduce the memory resources and also the processing time to unwrap the phase map. The weights are described in a typical matrix formulation allowing the matrix inversion using direct methods [14].

In this paper we give, in Section 2, the fundamentals of the 
phase unwrapping problem. We describe, in Section 3, our proposed phase unwrapping technique. In Section 4 some practical considerations to implement our algorithm are discussed. Numerical and real experiments are presented in Section 5 and finally, in Section 6, we summarize some conclusions.

\section{RELATIONSHIP BETWEEN WRAPPED AND UNWRAPPED PHASES}

Defining $\varphi_{r}$ and $\phi_{r}$ the wrapped and the unwrapped phase respectively, where $r=(x, y)$ is the vector in a discrete grid, the relationship between these two phases is established by

$$
\varphi_{r}=\mathcal{W}\left\{\phi_{r}\right\}=\phi_{r}+2 \pi k_{r}
$$

where $\mathcal{W}$ represents the wrapping operator and $k_{r}$ a field of integers such that $\mathcal{W}\left\{\phi_{r}\right\} \in(-\pi, \pi]$. The value of $\varphi_{r}$ represents the observed phase (wrapped) and $\phi_{r}$ the real unknown phase (unwrapped) to be determined. The discrete phase gradient field, $\nabla \varphi_{r}$, is defined as

$$
\nabla \varphi_{r}=\left(\varphi_{r}-\varphi_{s}, \varphi_{r}-\varphi_{t}\right)
$$

where $s=r-(1,0)$ and $t=r-(0,1)$ are contiguous horizontal and vertical sites respectively. We can also define the unwrapped discrete gradient field as $\nabla \phi_{r}=\left(\varphi_{r}-\phi_{s}, \phi_{r}-\phi_{t}\right)$. If the sampling theorem is fulfilled for these two discrete phase fields, the problem of recovering $\phi_{r}$ from $\varphi_{r}$ can be properly solved. The sampling theorem establishes that the distance between two fringes must be more than two pixels (the phase difference between two fringes is $2 \pi$ ). For phase map, the sampling theorem is reached if the phase difference between two pixels is less than $\pi$. This is

$$
\left\|\nabla \phi_{r}\right\|<\pi
$$

If this condition is satisfied, we can establish:

$$
\nabla \phi_{r}=\mathcal{W}\left\{\nabla \varphi_{r}\right\}=\left(\mathcal{W}\left\{\varphi_{r}-\varphi_{s}\right\}, \mathcal{W}\left\{\varphi_{r}-\varphi_{t}\right\}\right)
$$

$\mathcal{W}\left\{\nabla \varphi_{r}\right\}$ can be obtained from the observed field. From this equation, we see that $\phi_{r}$ can be achieved by two-dimensional integration of the vector field $\mathcal{W}\left\{\nabla \varphi_{r}\right\}$. This can be carried out by using a least-squares approach [15]-[17].

\subsection{Test for path independence}

Let $\Phi$ be a continuous vector field, then $\Phi$ has a pathindependent line integral if and only if

$$
\oint_{C \in V} \Phi(\mathbf{r}) \cdot \mathrm{d} \mathbf{r}=0
$$

$\Phi$ is a conservative vector field if there is a scalar function $\phi$ such that $\Phi=\nabla \phi_{r}$, for every piecewise simple closed curve $C$ in the domain of $\Phi$. Integrating $\nabla \phi_{r}$ around the path $r, s, u, t \in V, u=r-(1,1)$ (shown in Figure 1) then $q$ is expressed as,

$$
q=\sum_{i=1}^{4} \Delta_{i}
$$

where $\Delta_{1}=\varphi_{s}-\varphi_{r}, \Delta_{2}=\varphi_{u}-\varphi_{s}, \Delta_{3}=\varphi_{t}-\varphi_{u}$, $\Delta_{4}=\varphi_{r}-\varphi_{t}$. When $q$ is zero there are no inconsistencies.

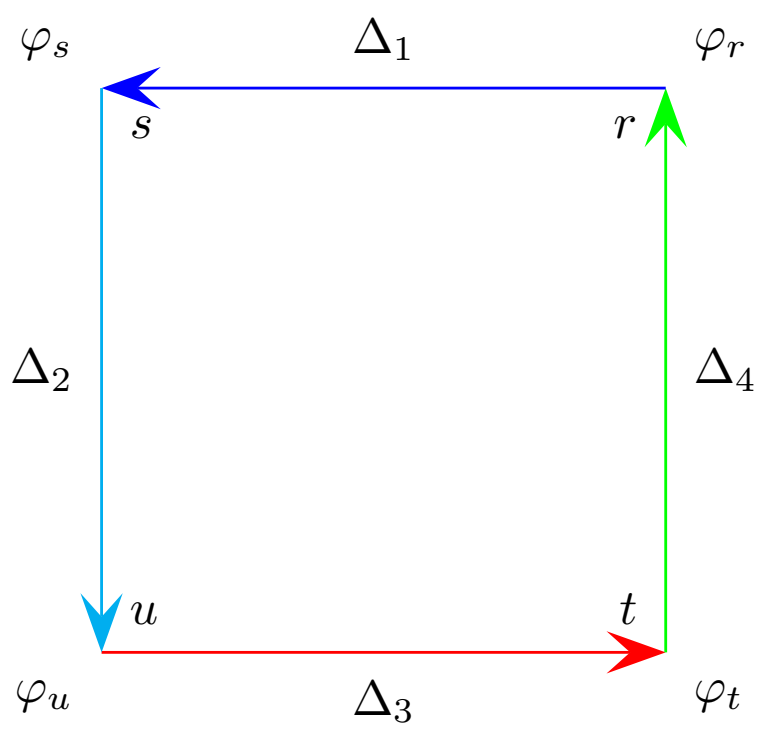

FIC. 1 Closed path $r, s, u, t \in V$ used for the path independence test.

\section{SAMPLED RADIAL BASIS FUNCTIONS FOR PHASE UNWRAPPING}

Any function can be modeled by a linear combination of basis functions. Let $U=\{0 \ldots M-1\} \subset \mathbb{Z}$ and $V=U \times U$ a smooth unwrapped phase $\phi: V \mapsto \mathbb{R}$ can be approximated by a linear combination of $N$ local RBF's, this is

$$
\phi_{r} \approx \sum_{i=1}^{N} w_{i} \psi\left(\left\|r-r_{i}\right\|\right)=\sum_{i=1}^{N} w_{i} \psi_{r}^{i},
$$

where, $r \in V, w_{i} \in \mathbb{R}(i \in\{1, \ldots, N\})$ are the weights of the $N$ shifted basis functions $\psi\left(\left\|r-r_{i}\right\|\right)=\psi_{r}^{i}$ and $r_{i} \in \mathbb{R}^{2}$ are the central point of the RBF's. Let $U_{h} \subset U$ and $U_{v} \subset U$ then, we take

$$
\left|U_{h}\right|,\left|U_{v}\right| \leq M
$$

to express the horizontal points $V_{h}=U_{h} \times U$ and vertical points $V_{v}=U \times U_{v}$ of the grid used to recover the phase.

The horizontal and vertical finite differences $\Delta_{s}^{h} \phi_{s}$ and $\Delta_{t}^{v} \phi_{t}$ are given by

$$
\begin{aligned}
\Delta_{s}^{h} \phi_{s} & =\phi_{s+\iota}-\phi_{s} \\
\Delta_{t}^{v} \phi_{t} & =\phi_{t+\kappa}-\phi_{t}
\end{aligned}
$$

where $s \in U_{h} \times\{0 \ldots M-2\}, t \in\{0 \ldots M-2\} \times U_{v}$, $\iota=(1,0), \kappa=(0,1)$. These finite differences can be expressed as:

$$
\begin{aligned}
& \Delta_{s}^{h} \phi_{s}=\Delta_{s}^{h} \sum_{i=1}^{N} w_{i} \psi_{s}^{i}=\sum_{i=1}^{N} w_{i} \Delta_{s}^{h} \psi_{s}^{i}=\sum_{i=1}^{N} w_{i} \Psi_{s}^{i} \\
& \Delta_{t}^{v} \phi_{t}=\Delta_{t}^{v} \sum_{i=1}^{N} w_{i} \psi_{t}^{i}=\sum_{i=1}^{N} w_{i} \Delta_{t}^{v} \psi_{t}^{i}=\sum_{i=1}^{N} w_{i} \Psi_{t}^{i}
\end{aligned}
$$

where $\Psi_{s}^{i}=\Delta_{s}^{h} \psi_{s}^{i}$ and $\Psi_{t}^{i}=\Delta_{t}^{v} \psi_{t}^{i}$. The phase unwrapping problem can be expressed in matrix form as

$$
\Phi_{d}=\Theta \mathbf{w}
$$


where $\Phi_{d}$ is the vector of unwrapped phase differences,

$$
\begin{gathered}
\Theta=\left(\begin{array}{ccccc}
\Theta_{h}^{1} & \Theta_{h}^{2} & \Theta_{h}^{3} & \ldots & \Theta_{h}^{N} \\
\Theta_{v}^{1} & \Theta_{v}^{2} & \Theta_{v}^{3} & \ldots & \Theta_{v}^{N}
\end{array}\right)=\left(\begin{array}{c}
\Theta_{h} \\
\Theta_{v}
\end{array}\right), \\
\mathbf{w}=\left(\begin{array}{c}
w_{1} \\
w_{2} \\
w_{3} \\
\vdots \\
w_{N}
\end{array}\right), \\
\Theta_{h}^{i}=\left(\begin{array}{c}
\Psi_{(k, 0)}^{i} \\
\Psi_{(k, 1)}^{i} \\
\Psi_{(k, 2)}^{i} \\
\vdots \\
\Psi_{(k, M-2)}^{i}
\end{array}\right) \Theta_{v}^{i}=\left(\begin{array}{c}
\Psi_{(0, l)}^{i} \\
\Psi_{(1, l)}^{i} \\
\Psi_{(2, l)}^{i} \\
\vdots \\
\Psi_{(M-2, l)}^{i}
\end{array}\right)
\end{gathered}
$$

and $k \in U_{h}, l \in U_{h}$.

For horizontal and vertical wrapped finite differences $\Delta_{S}^{h} \varphi_{S}$ and $\Delta_{t}^{v} \varphi_{t}$ (related to phase $\varphi$ ) we express

$$
\begin{aligned}
\mathcal{W}\left\{\Delta_{s}^{h} \varphi_{s}\right\} & =\mathcal{W}\left\{\varphi_{s+\iota}-\varphi_{s}\right\}=\varphi_{s}^{h} \\
\mathcal{W}\left\{\Delta_{t}^{v} \varphi_{t}\right\} & =\mathcal{W}\left\{\varphi_{t+\kappa}-\varphi_{t}\right\}=\varphi_{t}^{v}
\end{aligned}
$$

and using matrix notation:

$$
\begin{gathered}
\Omega_{h}=\left(\begin{array}{c}
\varphi_{(k, 0)} \\
\varphi_{(k, 1)} \\
\varphi_{(k, 2)} \\
\vdots \\
\varphi_{(k, M-2)}
\end{array}\right), \Omega_{v}=\left(\begin{array}{c}
\varphi_{(0, l)} \\
\varphi_{(1, l)} \\
\varphi_{(2, l)} \\
\vdots \\
\varphi_{(M-2, l)}
\end{array}\right) \\
\text { and } \Omega=\left(\begin{array}{c}
\Omega_{h} \\
\Omega_{v}
\end{array}\right)
\end{gathered}
$$

Given that $\mathcal{W}\left\{\Delta_{s}^{h} \varphi_{s}\right\}=\Delta_{s}^{h} \phi_{s}$ and $\mathcal{W}\left\{\Delta_{t}^{v} \varphi_{t}\right\}=\Delta_{t}^{v} \phi_{t}$, we can express the residual $\rho$ as

$$
\rho=\left(\begin{array}{c}
\Omega_{h} \\
\Omega_{v}
\end{array}\right)-\left(\begin{array}{c}
\Theta_{h} \\
\Theta_{v}
\end{array}\right) \mathbf{w}=\Omega-\Theta \mathbf{w}
$$

Minimizing the norm of the residual $\rho$

$$
\min _{\mathbf{w}}\|\rho\|=\min _{\mathbf{w}}\|\Omega-\hat{\Omega}\|^{2}=\min _{\mathbf{w}}\|\Omega-\Theta \mathbf{w}\|^{2}
$$

we obtain the optimal $\mathbf{w}^{*}$.

\section{SPATIAL DISTRIBUTION OF RBF'S FOR PHASE RECOVERING}

The distribution of the RBFs was chosen regular as shown in Figure 2. For distributions with $n \times n \mathrm{RBF}^{\prime}$, the width $\sigma$ for each RBF is given by

$$
\sigma=\frac{M}{(n-1) d_{s g}+2 d_{o}}
$$

where $M$ is the width of the wrapped phase, $d_{s g}$ is the distance between two nearest RBF's and $d_{0}$ is the distance of the RBF to

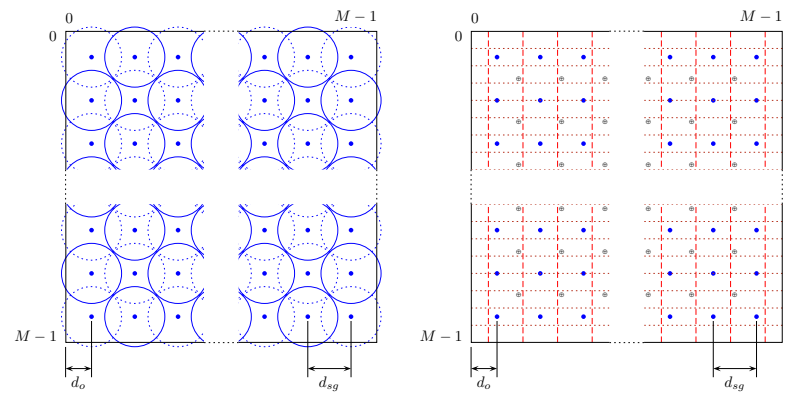

(a) Spatial distribution of RBF's centres (b) Distribution allowing the coverage with uncovered zones (distribution 1). of the whole phase Image (distribution 2). The grid of pixel lines, $V_{h} \cup V_{v}$, indicates the position of pixels used for the reconstruction.

FIG. 2 Regular distribution of RBF's within $V$. Their centres are represented by dots (or small circles) and their influence area by big circles.

the border of the image. Figure 2 shows the centre of the RBF's. The mesh in Figure 2(b) (red lines) shows a subset of pixels used in the reconstruction. It is important to take into account that there must be at least three horizontal and vertical pixel lines within the influence of the RBF's (blue circles) to achieve the reconstruction.

$$
G(\mathbf{x})=\exp \left(-\frac{\|\mathbf{x}\|^{2}}{2 \sigma^{2}}\right)
$$

where $\mathbf{x} \in V$.

\section{EXPERIMENTAL RESULTS}

In this section we present some experimental results from our proposed method and the methods reported by Villa et al. method (VM) [12] and Takeda et al (TM) [3]. All algorithms were evaluated with synthetic noiseless and synthetic noisy phase map. The first two algorithms were also applied to real wrapped phase maps. In all cases, our algorithm used two spatial distributions of RBF's (Figure 2). The centre of the RBF's is shown with dots. The coverage for each RBF for distribution 1 is shown by circles centred in each dot (Figure 2(a). Figure 2(b) only shows the centre of each RBF for distribution 2. Figure 3(a) shows a synthetic test phase map with $M=512$. Figures 3(b)-(c) show the noiseless and noisy wrapped phase maps, respectively, and Figure 4 shows their inconsistencies; inconsistencies in the noisy phase map are about 10 times bigger than those in the noiseless phase map. For comparison purposes all the reconstructed phase maps are wrapped. Figures 5(a)-(d) show the unwrapped synthetic phase maps from the corresponding to synthetic phase map (Figure 3(a)). The phase map shown in Figure 5(a) is obtained using VM, ( $\left.n=10, d_{s} g=1, d_{o}=0\right)$. The phase maps shown in Figure 5(c)-(d) are reconstructed using the proposed method with both distribution 1 (PMD1) and distribution 2 (PMD2) with $n=10, d_{s g}=1, d_{o}=0,\left|U_{v}\right|=\left|U_{v}\right|=50$, respectively. Finally, in Figure 5(d) we use a path dependent method proposed by TM. As show in Figures 6(a)-(b), the reconstructions using the VM and PMD1 are practically the same. The reconstruction error decreases when PMD2 is used (Figure 6(c) and TM reconstruction error is practically zero, Figure 6(d). In Ta- 


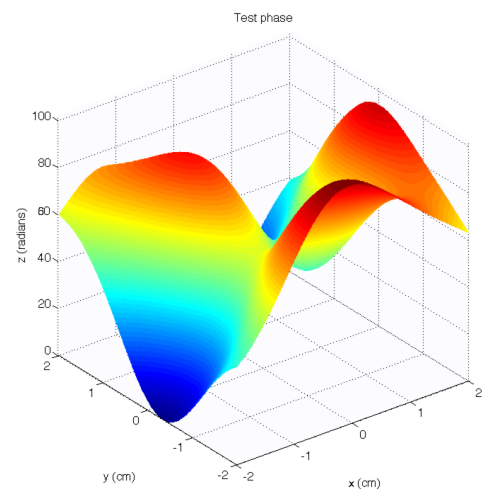

(a) Synthetic phase map.

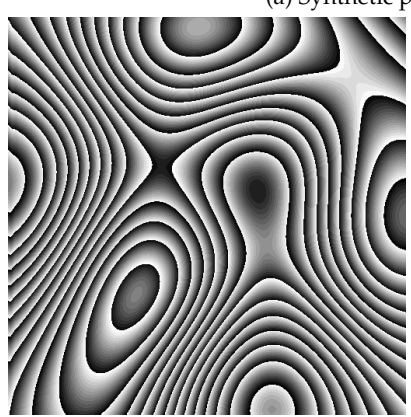

(b) Noiseless wrapped phase map.

FIG. 3 Wrapped test phase map.

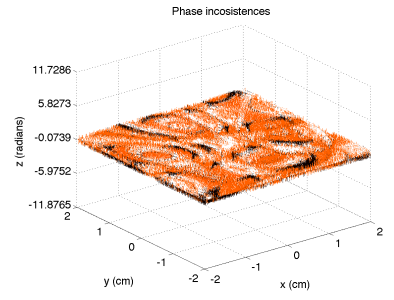

(a) Noiseless phase map inconsistencies.

FIG. 4 Inconsistencies in phase maps from Figure 3.

ble 1 is shown a summary of reconstruction time, memory and related error parameters. Unwrapped phase maps from wrapped noiseless phase map are shown in Figure 9.

In a second experiment the noisy wrapped phase map is used (shown in Figure 3(c)). The parameters used are $n=10$, $d_{s g}=1, d_{o}=0$ for $\mathrm{VM}$ and $n=10, d_{s g}=1, d_{o}=0$, $\left|U_{v}\right|=\left|U_{v}\right|=50$ for PMD1 and PMD2. The reconstruction quality for VM, PMD1 and PMD2 (Figures 9(a)-(c)) are higher than the TM (Figure 9(d)). The reconstruction errors are shown in Figure 10. In this case, the reconstruction errors for VM, PMD1 and PMD1 show a low error compared with TM. In Table 2 it is shown a summary of reconstruction time, memory and related error parameters. The reconstruction with PMD2 shows the lowest error but present greater memory usage and computing time than PMD2 and TM.

For real images we used two wrapped phase fields obtained experimentally, Figures 11(a) and 11(d). The first wrapped phase map has $M=235$ whereas the second wrapped phase map has $M=250$. We use $n=3$ for the first and $n=4$ for

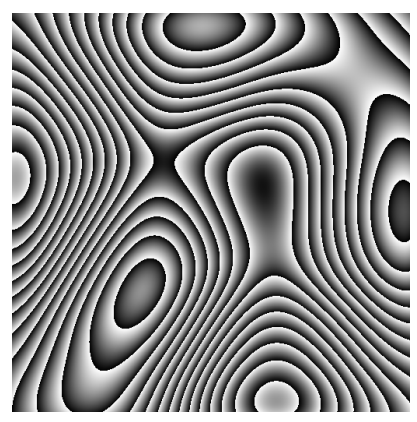

(a) Reconstruction using VM. $n=10$, $d_{s}=1, d_{0}=0$.

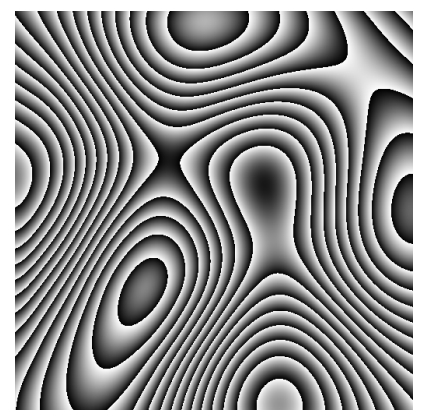

(c) Reconstruction using the proposed method, distribution 2. $n=10, d_{s}=1$, $d_{0}=0,\left|U_{v}\right|=\left|U_{v}\right|=50$.

FIG. 5 Wrapped recovered phase maps from wrapped noiseless phase map.

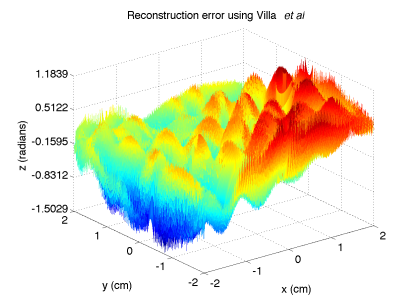

(a) Corresponding reconstruction error in Figure 5(a).

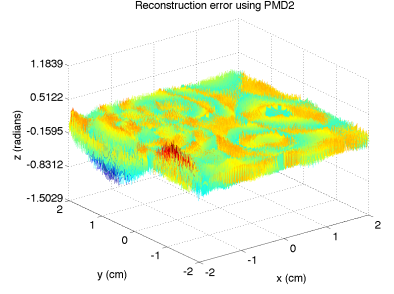

(c) Corresponding reconstruction error in Figure 5(c).

FIG. 6 Reconstruction error in the noiseless wrapped phase map shown in Figure 3 (b).

the second phase map in the phase unwrapping algorithm. In both cases we use the two distributions with $d_{s g}=1$ and $d_{0}=0$ and the space between lines of ten. The results applying our technique are shown in Figures 11(b), 11(c), 11(e) and 11(f).

We also applied our in a situation when there is a region of interest (ROI) or a pupil in the image (for example in optical tests). In this case we compare our method to Arines method [13]. From Figure 12 we can see that the reconstructed phase map for both methods are similar. For this case our method also presents better memory and time performance. 


\begin{tabular}{|l|r|c|c|c|c|r|}
\hline & \multirow{2}{*}{$\begin{array}{c}\text { Memory } \\
\text { Method }\end{array}$} & Time & \multicolumn{4}{|c|}{ Error } \\
\cline { 4 - 7 } & $(\mathrm{Kb})$ & $(\mathrm{seg})$ & $\mathrm{max}$ & $\mathrm{min}$ & rms & norm $_{2}$ \\
\hline \hline Villa et al. & 409600.000 & 6.011 & -1.503 & 1.184 & 0.0008 & 175.075 \\
\hline Proposed method $^{1}$ & 4096.000 & 0.694 & -1.449 & 1.205 & 0.0008 & 172.712 \\
\hline Proposed method $^{2}$ & 7413.760 & 1.492 & -0.795 & 0.670 & 0.0002 & 22.166 \\
\hline Takeda et al. & 4096.000 & 0.015 & -0.000 & -0.000 & 0.0000 & 0.000 \\
\hline
\end{tabular}

TABLE 1 Reconstruction performance and errors summary from Figure 6.

\begin{tabular}{|l|r|c|c|c|c|c|}
\hline & Memory & Time & \multicolumn{4}{|c|}{ Error } \\
\cline { 4 - 7 } Method & $(\mathrm{Kb})$ & $(\mathrm{seg})$ & $\max$ & \multicolumn{1}{c|}{$\min$} & \multicolumn{1}{c|}{ rms } & \multicolumn{1}{c|}{ norm $_{2}$} \\
\hline \hline Villa et al. & 409600.000 & 6.110 & -7.981 & 6.274 & 0.0057 & 1111.441 \\
\hline Proposed method $^{1}$ & 4096.000 & 0.698 & -7.706 & 6.297 & 0.0057 & 1205.877 \\
\hline Proposed method $^{2}$ & 7413.760 & 1.455 & -7.168 & 6.225 & 0.0054 & 1095.579 \\
\hline Takeda et al. & 4096.000 & 0.022 & -46.916 & 39.179 & 0.0263 & 6394.984 \\
\hline
\end{tabular}

TABLE 2 Reconstruction performance and errors summary from Figure 10.

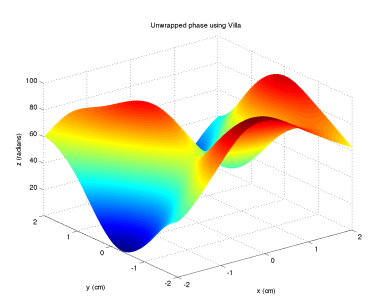

(a) Reconstruction using VM. $n=10$ $d_{s}=1, d_{0}=0$.

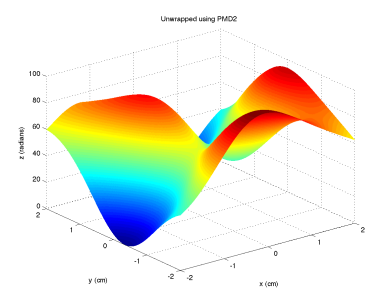

(c) Reconstruction using the proposed method, distribution 2. $n=10, d_{s}=1$, $d_{0}=0,\left|U_{v}\right|=\mid U_{v}=50$.

FIC. 7 Unwrapped phase maps from the wrapped noiseless phase map.

\section{CONCLUSIONS}

We have presented an algorithm based on sampled radial basis functions to recover the phase from a wrapped phase map. This algorithm has good performance when working with smooth phase maps with low level of noise. The sampling of the phase map allows to decrease both the processing time and the memory resource required to unwrapped the phase when compared to the VM and Arines methods [13]. We found that The distribution 2 produces better results than distribution 1 when is it applied to a noisy phase map and to an interferograms with pupil.

\section{ACKNOWLEDGMENTS}

We want to thank the Universidad Autónoma de Zacatecas, the National Institute of Astrophysics, Optics and Electronics, and

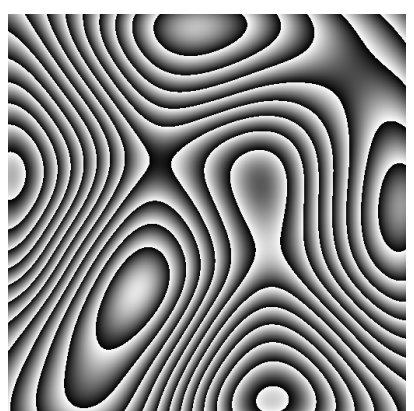

(a) Reconstruction using VM. $n=10$, $d_{s}=1, d_{0}=0$.

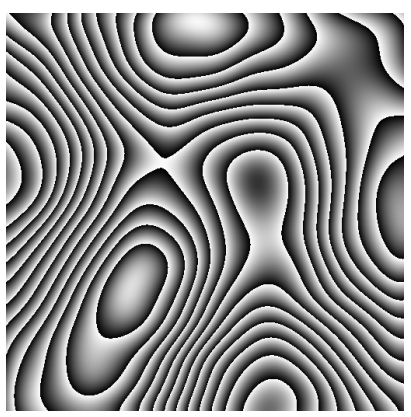

(c) Reconstruction using the proposed method, distribution 2. $n=10, d_{s}=1$, $d_{0}=0,\left|U_{v}\right|=\left|U_{v}\right|=50$.

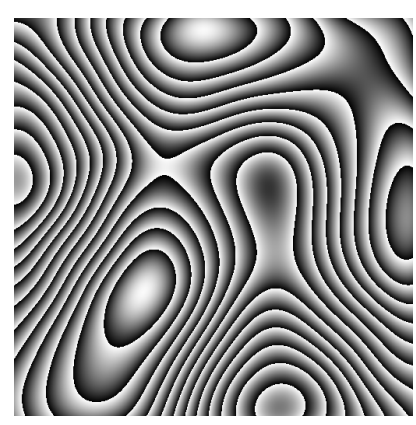

(b) Reconstruction using the proposed method, distribution $1 . n=10, d_{s}=1$, $d_{0}=0,\left|U_{v}\right|=\left|U_{v}\right|=50$.

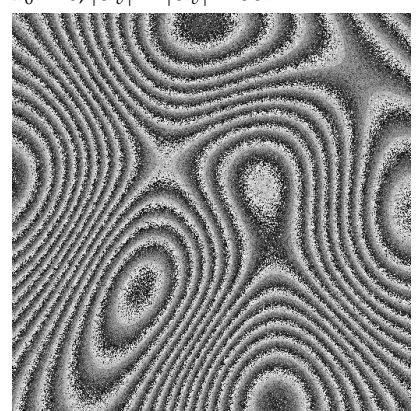

(d) Reconstruction using TM.
FIG. 8 Wrapped recovered phase maps from the wrapped noisy phase map.

PIFI-2013-32MSU0017H-09 DES ingeniería for all the support for this work.

\section{References}

[1] C. M. Vest, Holographic Interferometry (John Wiley at Sons, New York, 1979).

[2] K. J. Gasvik, Optical Metrology (Wiley, New York, 1987).

[3] M. Takeda, H. Ina, and S. Kobayashi, "Fourier-Transform Method of Fringe-Pattern Analysis for Computer-Based Topography and Interferometry," J. Opt. Soc. Am. A 72, 156-159 (1981).

[4] D. Malacara, M. Servín, and Z. Malacara, Interferogram Analysis for Optical Testing (Marcel Dekker, New York, 1998). 


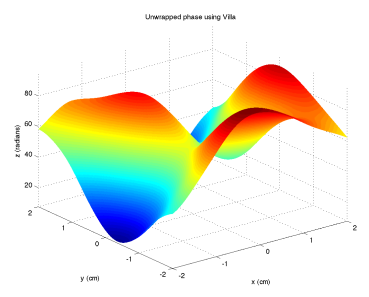

(a) Reconstruction using VM. $n=10$, $d_{\mathrm{s}}=1, d_{0}=0$.

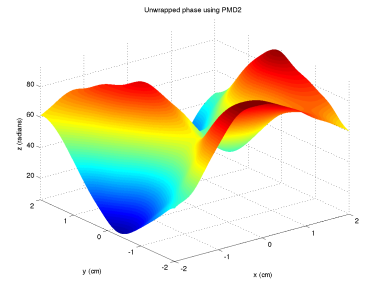

(c) Reconstruction using the proposed method, distribution 2. $n=10, d_{s}=1$, $d_{0}=0,\left|U_{v}\right|=\left|U_{v}\right|=50$.

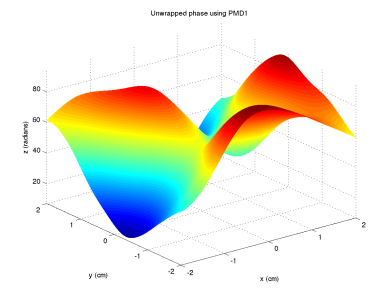

(b) Reconstruction using the proposed method, distribution 1. $n=10, d_{s}=1$ $d_{0}=0,\left|U_{v}\right|=\left|U_{v}\right|=50$.

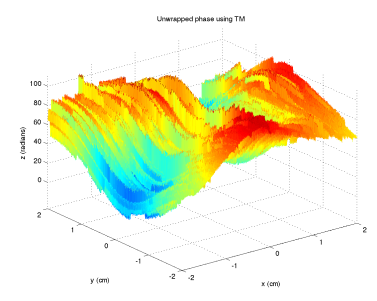

(d) Reconstruction using TM.

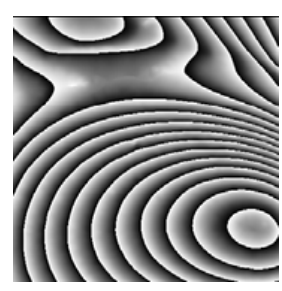

(a) Interferogram one.

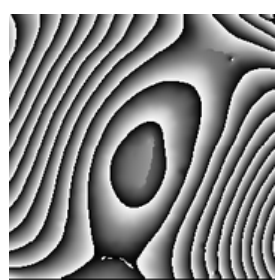

(d) Interferogram two

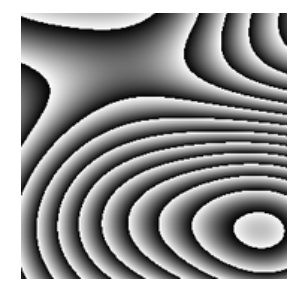

(b) Wrapped phase of the phase map recovered using distribution 1.

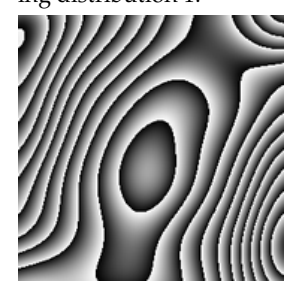

(e) Wrapped phase of the phase map recovered using distribution 1.

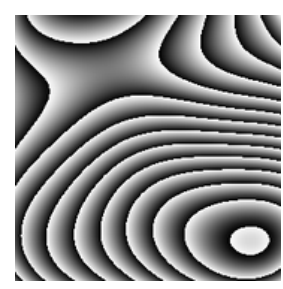

(c) Wrapped phase of the phase map recovered using distribution 2

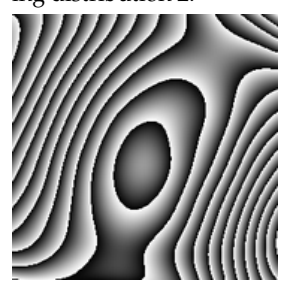

(f) Wrapped phase of the phase map recovered using distribution 2 .

FIG. 9 Unwrapped phase maps from the wrapped noisy phase map.

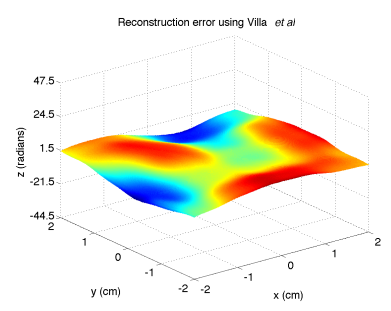

(a) Corresponding reconstruction error in Figure 5(a).

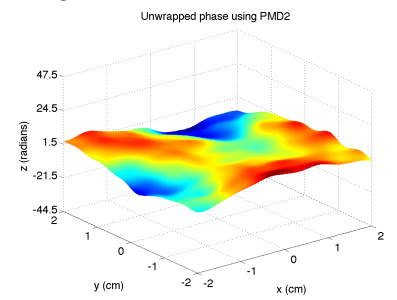

(c) Corresponding reconstruction error in Figure 5(c).

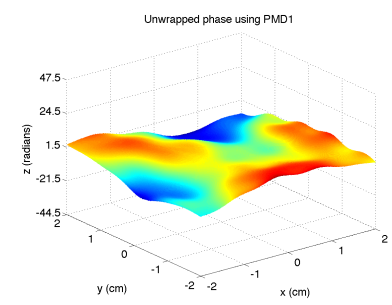

(b) Corresponding reconstruction error in Figure 5(b).

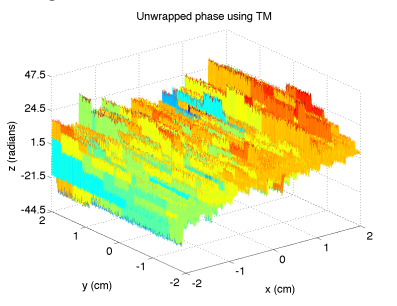

(d) Corresponding reconstruction error in Figure 5(d).

FIG. 10 Reconstruction errors in the noisy wrapped phase map shown in Figure 3(c).

[5] J. Villa, I. de la Rosa, G. Miramontes, and J. A. Quiroga, "Phase recovery from a single fringe pattern using an orientational vector field regularized estimator," J. Opt. Soc. Am. A, 22, 2766-2773 (2005).

[6] L. Guerriero, G. Nico, G. Pasquariello, and S. Stramaglia, "New regularization scheme for phase unwrapping," Appl. Optics 37(14), 3053-3058 (1998).

[7] M. Rivera and J. L. Marroquin, "Half-quadratic cost functions for phase unwrapping," Opt. Lett. 29(5), 504-506 (2004).

[8] B. Ströbel, "Processing of interferometric phase maps as complexvalued phasor images," Appl. Optics 35, 2192-2198 (1996).

[9] D.C. Ghiglia, and M.D. Pritt, Two-dimensional phase unwrapping: theory, algorithms, and software (Wiley, New York, 1998).
FIG. 11 Proposed algorithm applied to two real interferograms.

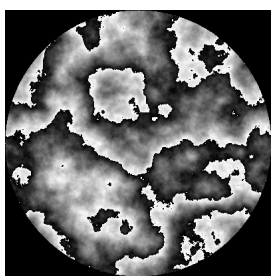

(a) Phase map

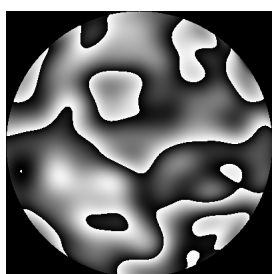

(b) Wrapped phase of the phase map recovered using distributions 2

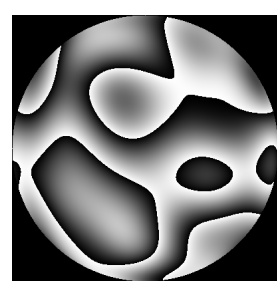

(c) Wrapped phase of ered using the Arines' method [13] the phase map recov-

FIG. 12 Interferogram with pupil.

[10] B. R. Hunt, "Matrix formulation of the reconstruction of phase values from phase differences," J. Opt. Soc. Am. 69(3), 393-399 (1979).

[11] D. C. Chiglia, and L. A. Romero, "Robust Two-Dimensional Weighted and Unweighted, Phase Unwrapping for Uses Fast Transform and Iterative Methods," J. Opt. Soc. Am. A 11, 107-117 (1994).

[12] J. Villa Hernández, I. de la Rosa Vargas, and Enrique de la Rosa Miranda, "Radial Basis Functions for Phase Unwrapping," Computación y Sistemas 14, 145-150 (2009).

[13] J. Arines, "Least-squares modal estimation of wrapped phases: Application to phase unwrapping," Appl. Optics 42(17), 3373-3378 (2003).

[14] G. H. Golub, and C. F. Van Loan, Matrix Computations (The John Hopkins University Press, Maryland, 1996).

[15] V. Lyuboshenko, H. Mâtre, and A. Maruani, "Least-Mean-Squares Phase Unwrapping by Use of an Incomplete Set of Residue Branch Cuts," Appl. Optics 41, 2129-2148 (2002).

[16] Y. Lu, X. Wang, and X. Zhang, "Weighted least-squares phase unwrapping algorithm based on derivative variance correlation map," Optik 118(2), 62-66 (2007).

[17] S. B. Kim, and Y. S. Kim, "Least Squares Phase Unwrapping in Wavelet Domain," IEE P.-Vis. Image Sign. 152(3), 261-267 (2005). 